\title{
Rapidly Deteriorating Dysphagia Complicating Steroid - Resistant Dermatomyositis Successfully Treated with Intravenous Immunoglobulin
}

\author{
Muhammad Abdur Rahim¹, Hasna Fahmima Haque ${ }^{2}$, Pratik Dewan ${ }^{3}$, AKM Shaheen Ahmed ${ }^{4}$, Khwaja Nazim
} Uddin $^{5}$.

\begin{abstract}
Dermatomyositis typically presents with proximal myopathy and skin rash. Dysphagia is not an uncommon feature. Here we describe a middle aged Bangladeshi man, who presented with facial puffiness and erythema, photosensitivity, polyarthritis and proximal myopathy. Diagnostic work-up confirmed dermatomyositis. Malignancy and other connective tissue diseases were excluded. The patient did not respond to conventional immunosuppressive agents, rather deterioration occurred to involve pharyngeal and respiratory muscles. He required transfer to intensive care unit, mechanical ventilation and tracheostomy. His symptoms improved with intravenous-immunoglobulin.
\end{abstract}

Key words: dermatomyositis, dysphagia, intravenous-immunoglobulin.

\section{Introduction}

Dermatomyositis is a variety of primary inflammatory myositis that typically occurs in 40-60 years age group and twice more commonly among females. Common presentations include proximal weakness, muscle tenderness and characteristic rash. Dysphagia can occur in up to three-fourth of patients and carries a poor prognosis. ${ }^{1-3}$ Respiratory muscle involvement needs mechanical ventilation and aggressive immunosuppressive treatment. ${ }^{4,5}$ Adult-onset dermatomyositis is often associated with an underlying malignancy and care should be taken to exclude associated connective tissue disease. This case is reported here to highlight the role of intravenous-immunoglobulin (IVIg) in treatment of steroid-resistant dermatomyositis complicated with dysphagia.

1. Dr. Muhammad Abdur Rahim, FCPS (Med.), Assistant Professor, Department of Nephrology, BIRDEM General Hospital, Shahbag, Dhaka, Bangladesh.

2. Dr. Hasna Fahmima Haque, FCPS (Med.), Registrar, Department of Internal Medicine, BIRDEM General Hospital, Shahbag, Dhaka, Bangladesh.

3. Dr. Pratik Dewan, MD (Int. Med.), Associate Consultant, Department of Internal Medicine and Endocrinology, Square Hospitals Ltd, Dhaka, Bangladesh.

4. Dr. AKM Shaheen Ahmed, FCPS (Med.), MCPS (Med.), Associate Professor, Department of Internal Medicine, BIRDEM General Hospital, Shahbag, Dhaka, Bangladesh.

5. Dr. Khwaja Nazim Uddin, FCPS (Med.), FACP, FRCS, Professor, Department of Internal Medicine, BIRDEM General Hospital, Shahbag, Dhaka, Bangladesh.

Corresponding author:

Dr. Muhammad Abdur Rahim, FCPS (Medicine)

Assistant Professor, Department of Nephrology

BIRDEM General Hospital

Shahbag, Dhaka-1000, Bangladesh

Email: muradrahim23@yahoo.com.

\section{Case report}

A 48-year-old Bangladeshi gentle man presented with progressively increasing periorbital puffiness and redness and photosensitivity over the face for two and half months, arthritis of hand joints for 15 days, tightening and swelling of skin of the forearms, hands and thighs and proximal limb weakness for 12 days without any fever, Raynaud's phenomenon and difficulty in swallowing. He did not have anorexia or night sweats. He lost $2 \mathrm{~kg}$ in weight over this period. He was not abusing alcohol nor on any medication.

His face looked puffy and erythematous. His skin over face, fore arms, hands and thighs was shiny, taught and indurated. There were few painful, palpable purpura in fingers, legs and toes (Figure 1). He was not anaemic and there was no lymphadenopathy. His metacarpophalangeal and proximal interphalangeal joints of both hands were swollen and tender. Muscle power specially in proximal groups was reduced (upper limb 3/5, lower limb 3/5). Deep tendon reflexes and plantar responses were normal. Other examination findings were unremarkable.

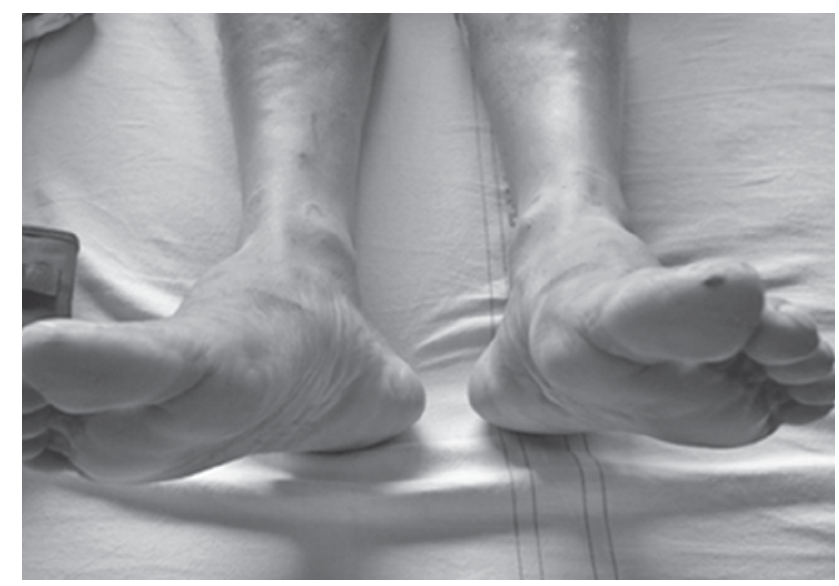

Figure 1. Purpura on legs and toes 
$\mathrm{His} \mathrm{Hb} \%$, total and differential white blood cells and platelet counts were normal. ESR was $110 \mathrm{~mm}$ in $1^{\text {st }}$ hour, CRP was positive. Urine routine examination did not show any red cell, cast or protein. Hepatic transaminases (ALT $267 \mathrm{U} / \mathrm{L}$, AST $657 \mathrm{U} / \mathrm{L})$, creatine phosphokinase (CPK) (44,540 U/L, normal 29-195), lactate dehydrogenase (LDH) (2928 U/L, normal 230-460) and aldolase (23 U/L, normal up to 7.6) were markedly raised. Antinuclear antibody (ANA) was positive but none of the extractable nuclear antibodies (ENA) was positive. Anti-neutrophil cytoplasmic antibodies (ANCAs) were also negative. Serum bilirubin, electrolytes, thyroid function, chest X-ray and abdominal ultrasound were normal.

Electromyography (EMG) was suggestive of inflammatory muscle disease (myositis). Histopathology of left calf muscles confirmed the diagnosis of dermatomyositis (Figure 2). CT scan of chest and abdomen and upper GI endoscopy were normal as were tumour markers.

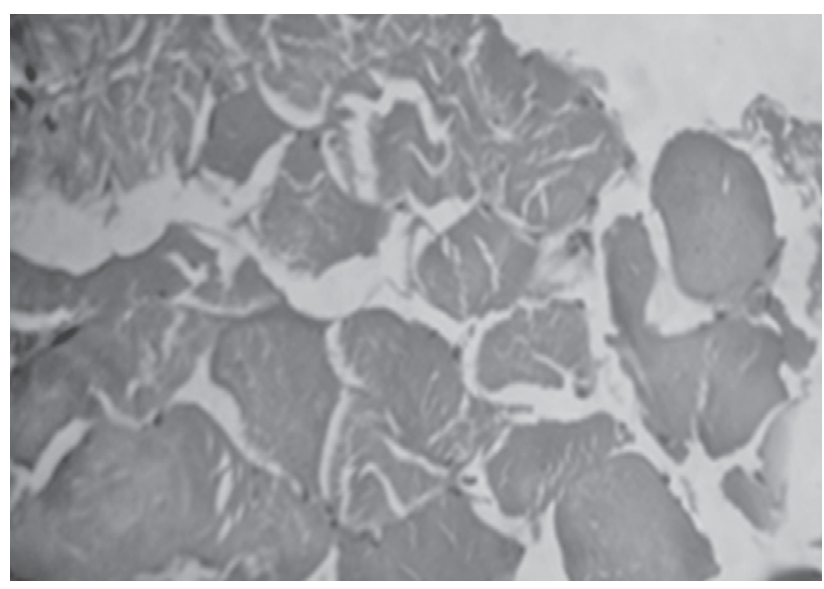

Figure 2. Histopathological slide from calf muscles showing mononeuclear cellular infiltration suggesting inflammatory myositis

Treatment was started with prednisolone $1 \mathrm{mg} / \mathrm{kg} / \mathrm{day}$ and azathioprine was added later on. Subcutaneous methotrexate was initiated because of poor response to prednisolone and azathioprine. But his response to medication was not good, rather weakness involved his neck muscles and he was unable to hold his neck in upright position and developed swallowing difficulty. Intravenous methyl prednisolone was added at a dose of $1 \mathrm{gm} /$ day for 3 days without any benefit. As a precautionary measure, for the possibility of respiratory muscle involvement and respiratory failure the patient was shifted to intensive care unit (ICU).

At ICU endotracheal intubation was done for protection of air way and IVIg was started at a dose of $0.4 \mathrm{gm} / \mathrm{kg} / \mathrm{day}$ and continued for 3 days. His muscle power improved to $4 / 5$, but swallowing difficulty persisted. Tracheostomy was done as he was supposed to require long term endotracheal intubation for airway protection.

Two weeks later he was shifted to general ward with tracheostomy tube and nasogastric (NG) feeding tube in situ. After another one week, his biochemical and inflammatory parameters showed significant improvement (CPK 329 U/L,
AST $94 \mathrm{U} / 1$, ESR $54 \mathrm{~mm}$ in $1^{\text {st }}$ hour). His discharge medications included methotrexate $10 \mathrm{mg}$ subcutaneously weekly and prednisolone $0.75 \mathrm{mg} / \mathrm{kg} /$ day along with folic acid, calcium and alendronate.

Subsequent follow up visits at $2^{\text {nd }}, 5^{\text {th }}$ and $8^{\text {th }}$ weeks showed marked clinical and biochemical improvements. He can take food orally, NG tube was removed and closure of tracheostomy wound was done at $6^{\text {th }}$ week post-discharge.

\section{Discussion}

Diagnosis of dermatomyositis is straight-forward. In a patient with typical clinical presentation, demonstration of elevated muscle enzymes, EMG and muscle biopsy findings are sufficient to make a diagnosis. Drug response is often unsatisfactory in patients with dermatomyositis having dysphagia. Such patients need prolonged NG feeding or percutaneous gastrostomy and aggressive immunosuppressive therapy. ${ }^{6-8}$ Respiratory paralysis and failure in dermatomyositis require endotracheal intubation and mechanical ventilation. ${ }^{5}$ In few patients, non-invasive ventilations are of benefit. ${ }^{4}$ On rare occasions, tracheostomy appears live saving. ${ }^{9}$

One-third of adult onset dermatomyositis is associated with an occult malignancy, especially colorectal, bladder, lung, non-Hodgkin's lymphoma, ovarian, pancreatic and stomach cancers. If not found, their risk for developing cancer remains for up to five years. Some adult patients may have associated arthritis, Raynaud's etc. In such patients an overlap syndrome or mixed connective tissue disease should be excluded. ${ }^{10}$

Corticosteroids are the mainstay of treatment. Resistant dermatomyositis is the term used to describe when disease does not respond to conventional immunosuppressive treatment. Such cases often need combination of immunosuppressive drugs, IVIg, anti-TNF or other agents. ${ }^{11-14}$ Response is variable but most reports are in favour of IVIg. Our patient initially did not respond to prednisolone, azathioprine, IV pulse methyl prednisolone but rapidly responded to IVIg. Now he is on subcutaneous methotrexate and tapering doses of prednisolone.

\section{Acknowledgement}

We express our acknowledgement to Prof. Syed Atiqul Haq, Chairman, Rheumatology Wing of BSMMU and all ICU Consultants and stuffs of BIRDEM for their assistance and advice in managing the case.

\section{References}

1. Oh T, Brumfield K, Hoskin T, Stolp KA, Murray JA, Bassford JR. Dysphagia in inflammatory myopathy: clinical characteristics, treatment strategies and outcome in 62 patients. Mayo Clinic Proceedings 2007; 82(4):441-47.

2. Marie I. Morbidity and mortality in adult polymyositis and dermatomyositis. Curr Rheumatol Rep 2012;14(3):275-85.

3. Williams R, Grehan M, Hersch M, Andre J, Cook I. Biomechanics, diagnosis and treatment outcome in inflammatory myopathies presenting as oropharyngeal dysphagia. Gut 2003; 52:471-78. 
4. Azuara BO, Cía IG, Polo CS. Non-invasive mechanical ventilation in intensive care unit in patient with respiratory failure as the first manifestation of dermatomyositis. An Med Interna 2005;22(9):434-36.

5. Salimbene I, Leli I, Valente S. Respiratory failure in a patient with dermatomyositis. Multidiscip Respir Med 2013; 8(1): 27.

6. Marie I, Hachulla E, Levesque H, Reumont G, Ducrotte P, Cailleux $\mathrm{N}$ et al. Intravenous immunoglobulins as treatment of life threatening esophageal involvement in polymyositis and dermatomyositis. J Rheumatol 1999;26(12):2706-9.

7. Zuber MA, Kouba M, Rudolph SE, Weller M, Hrdlicka P. Severe dysphagia and erythrodermia in a 59-year-old man. Internist (Berl) 2013;54(3):359-65

8. Marie I, Menard JF, Hatron PY, Hachulla E, Mouthon L, Tiev K et al. Intravenous immunoglobulins for steroid-refractory esophageal involvement related to polymyositis and dermatomyositis: a series of 73 patients. Arthritis Care Res (Hoboken) 2010; 62(12): 1748-55.

9. Iester A, Puleo MG, Buzzanca C, Debbia C, Callegarini L, Alpigiani MG. A case of organic psychosis in dermatomyositis. Etiopathogenetic hypothesis. Minerva Pediatr 1994;46(12):565-68.
10. Andrews J, Hall MA. Dermatomyositis-scleroderma overlap syndrome presenting as autoimmune haemolytic anaemia. Rheumatology (Oxford) 2002;41(8):956-58.

11. Cagnoli M, Marchesoni A, Tosi S. Combined steroid, methotrexate and chlorambucil therapy for steroid-resistant dermatomyositis. Clin Exp Rheumatol 1991;9(6):658-59.

12. Murota H, Muroi E, Yamaoka T, Hamasaki Y, Katayama I. Successful treatment with regimen of intravenous gamma globulin and cyclophosphamide for dermatomyositis accompanied by interstitial pneumonia, opportunistic infection and steroid psychosis. Allergol Int 2006;55(2):199-202.

13. Efthimiou P, Schwartzman S, Kagen LJ. Possible role for tumour necrosis factor inhibitors in the treatment of resistant dermatomyositis and polymyositis: a retrospective study of eight patients. Ann Rheum Dis 2006; 65(9): 1233-36.

14. Dalakas MC. Therapeutic Advances and Future Prospects in Immune-Mediated Inflammatory Myopathies. Ther Adv Neurol Disord 2008; 1(3): 157-66. 